\title{
VASA PREVIA, PRESENTACIÓN DE UN CASO EN UN CENTRO DE REFERENCIA EN ENVIGADO, COLOMBIA, Y REVISIÓN DE LA LITERATURA
}

\section{Vasa previa, case presentation from a referral center in Envigado, Colombia, and review of the literature}

Óscar Alejandro Bonilla-Sepúlveda, $M D^{1}$

Recibido: julio 29/13 - Aceptado: marzo 11/14

\section{RESUMEN}

Objetivo: reportar un caso de vasa previa y hacer una revisión de la literatura en cuanto a la patogénesis, el diagnóstico ecográfico antenatal y el pronóstico perinatal de la misma.

Materiales y métodos: se presenta el caso de una paciente de 44 años de edad y 36 semanas de gestación, que consultó al Hospital Manuel Uribe Ángel (HMUA), institución pública de segundo nivel de atención, centro de referencia obstétrica ubicado en el municipio de Envigado, Antioquia. Se utilizó como fuente de información la historia clínica, se realizó una búsqueda de literatura con las palabras clave "vasa previa", "ultrasonography", "prognosis" en las bases de datos Medline vía PubMed de los años 1993 a 2013 en inglés y español; se buscaron revisiones de tema, revisiones sistemáticas y reportes de caso.

Resultados: se encontraron 35 artículos, se analizaron todas las referencias y se halló que 22 corresponden a reportes de caso, 8 a revisiones de tema y 5 a revisiones sistemáticas.

1 Médico ginecoobstetra, Universidad CES, Medellín, Colombia. oalejobonilla@yahoo.es
Conclusiones: la vasa previa es una complicación obstétrica poco frecuente. La evaluación de la placenta por ultrasonido es un método útil para determinar la inserción del cordón umbilical y sus posibles anormalidades. El pronóstico es pobre, con altos índices de mortalidad fetal si no se realiza un diagnóstico antenatal.

Palabras clave: vasa previa, ultrasonografía, pronóstico.

\section{ABSTRACT}

Objective: To report a case of vasa previa and a review of the literature in terms of the pathogenesis, pre-natal ultrasound diagnosis, and perinatal prognosis.

Materials and methods: We present a case of a 44 year-old woman in her $36^{\text {th }}$ week of gestation who came to Hospital Manuel Uribe Ángel (HMUA), a level II public referral center in Envigado, Antioquia. The clinical record was used as a source of information. The search of the literature was conducted using the key words vasa previa, ultrasonography, prognosis in the Medline databases through PubMed between 1993 and 2013, in English and Spanish. The search focused on topic reviews, systematic reviews, and case reports. 
Results: Overall, 35 articles were found, all the references were analyzed, resulting in 22 case reports, 8 topic reviews, and 5 systematic reviews. Conclusions: Vasa previa is an infrequent obstetrical complication. Ultrasound placental assessment is a useful tool to determine umbilical cord insertion and potential abnormalities. Prognosis is poor, with high rates of fetal mortality in the absence of pre-natal diagnosis.

Key words: Vasa previa, ultrasonography, prognosis.

\section{INTRODUCCIÓN}

La vasa previa se define como la presencia de vasos sanguíneos fetales o placentarios que discurren por las membranas ovulares en una porción baja del segmento uterino, cubriendo el orificio cervical interno y anterior a la presentación fetal (1). Estos vasos han sido considerados como anormales porque se ubican en las membranas amnióticas sin tener gelatina de Wharton o tejido placentario, y apenas con un pliegue de amnios, lo que los hace propensos a la ruptura en caso de amniotomía o ruptura espontánea (2). Se clasifican en tipo I -llamado también inserción velamentosa del cordón- cuando el cordón umbilical se inserta en las membranas ovulares en lugar de hacerlo en la placenta, y tipo II cuando los vasos sanguíneos cruzan entre la placenta y un lóbulo placentario accesorio (3). La prevalencia informada varía entre 1,5-4:10.000 nacidos vivos (3). Lobstein reportó el primer caso de la rotura de vasa previa en 1801 (4), y la primera descripción de ultrasonido en vasa previa se remonta a 1987 (5). Antes del ultrasonido el diagnóstico de vasa previa se hacía tardíamente, y se expresaba con la tríada: ruptura de membranas, sangrado vaginal indoloro y sufrimiento fetal (hemorragia de Benckiser).

Es difícil realizar el diagnóstico clínico anteparto a menos que se presente con una hemorragia del segundo o tercer trimestre, o más frecuente cuando se da la ruptura espontánea o artificial de membranas (6). Se presenta como un sangrado vaginal inmediato a la ruptura de membranas, asociado a cambios cardiovasculares agudos en el feto por anemia, y se manifiesta con ritmo sinusoidal en el monitoreo fetal, bradicardia sostenida o la muerte por exanguinación (7-10). La mortalidad fetal informada para los casos no reconocidos antes del parto varía entre el 22,5 y $100 \%$ (11), por ello todos los esfuerzos están dirigidos al diagnóstico prenatal.

Se ha descrito la utilidad de diferentes tecnologías para el diagnóstico, de las cuales hay reportes de la amnioscopia $(12,13)$, pero este examen necesita un entrenamiento especial en un hospital de referencia, sus costos son elevados, presenta limitaciones en el diagnóstico y complicaciones secundarias al procedimiento. Otros autores se han inclinado por pruebas de laboratorio clínico en aquellos casos en los cuales se presenta sangrado vaginal materno e indican la electroforesis de proteínas para identificar hemoglobina fetal o detectar glóbulos rojos nucleados que son más frecuentes de encontrar en la sangre fetal que en la materna (Test Ogita) (14); sin embargo, esta prueba no tiene ninguna utilidad en situación de emergencia por sangrado materno que comprometa la vida, y presenta muchos falsos positivos y negativos (15-18). La resonancia magnética nuclear también se ha usado para el diagnóstico pero es impráctica por su alto costo (19). En la actualidad, la ecografía con doppler color ofrece ventajas por ser no invasiva y de bajo costo $(5,20)$, no obstante, es poco lo que se conoce sobre su exactitud.

Dada la baja frecuencia de la vasa previa, los retos diagnósticos que representa para el ginecólogo y la importancia de tenerla en cuenta dentro del diagnóstico del sangrado del segundo y tercer trimestres, se presenta este caso con el objetivo de hacer una revisión de la literatura publicada respecto a la exactitud del ultrasonido para su diagnóstico, así como también revisar la patogénesis y el pronóstico de esta patología.

\section{REPORTE DE CASO}

Paciente de 44 años, grávida: 3, paridad: 2, aborto: 0 (G3P2A0); fecha de último parto, 15 de diciembre de 1999; raza mestiza; con secundaria incompleta, casada. Previo al embarazo consumía 8 cigarrillos 
al día, niega alcohol u otras drogas. En el examen físico presenta un peso de $58 \mathrm{~kg}$ y talla de $1,65 \mathrm{~m}$. El control prenatal ha sido adecuado (5 asistencias), hemo-clasificación, $\mathrm{O}+$; serología no reactiva, hemoglobina de 13,2 g/dl, citología negativa para lesión intraepitelial escamosa (LIE), mamas y odontológico normales, antitetánica vigente; toma hierro y folato, con una ganancia de peso total de 9,5 kg, promedio presiones arteriales 100/70 mm/ hg. Le habían realizado 3 ecografías: una de ingreso al control prenatal a la semana $12+1$, normal; la segunda a las $22+4$ semanas creciendo en percentil 55, placenta corporal anterior, y la última a la semana $28+3$ creciendo en percentil 50 simétrico, reporta placenta anterior, madurez grado 1 , líquido amniótico adecuado y placenta con lóbulo placentario accesorio posterior pequeño, por lo cual fue remitida para evaluación al servicio de ultrasonido del Hospital Manuel Uribe Ángel (HMUA), institución pública de segundo nivel de complejidad, que atiende pacientes de estrato socioeconómico medio y bajo, ubicado en el municipio de Envigado, departamento de Antioquia, en marzo de 2012 para realización de ultrasonido, en el que se encontró placenta anterior con madurez grado 2, con lóbulo placentario accesorio; se complementa con ecografía transvaginal y doppler color encontrando que los vasos sanguíneos están en una posición fija entre la placenta anterior y el lóbulo accesorio posterior (figura 1). Es diagnosticada como vasa previa tipo II y se recomendó control prenatal por alto riesgo obstétrico y seguimiento ecográfico, los controles prenatales se consideraron normales, pero no se realizaron más controles ecográficos hasta el ingreso por urgencias al HMUA con 36 semanas de gestación, sangrado vaginal severo, indoloro, sin aumento en el tono uterino y con disminución de los movimientos fetales. Requirió realizar cesárea de urgencia, se obtuvo feto masculino, vivo, pero con puntaje de APGAR de 4 al minuto y 6 a los cinco minutos, necesitó reanimación neonatal básica por pediatría, con control de hemoglobina fetal $6,5 \mathrm{~g} / \mathrm{dl}$ que requirió transfusión y permaneció hospitalizado 4 días en la unidad de cuidados intensivos neonata-

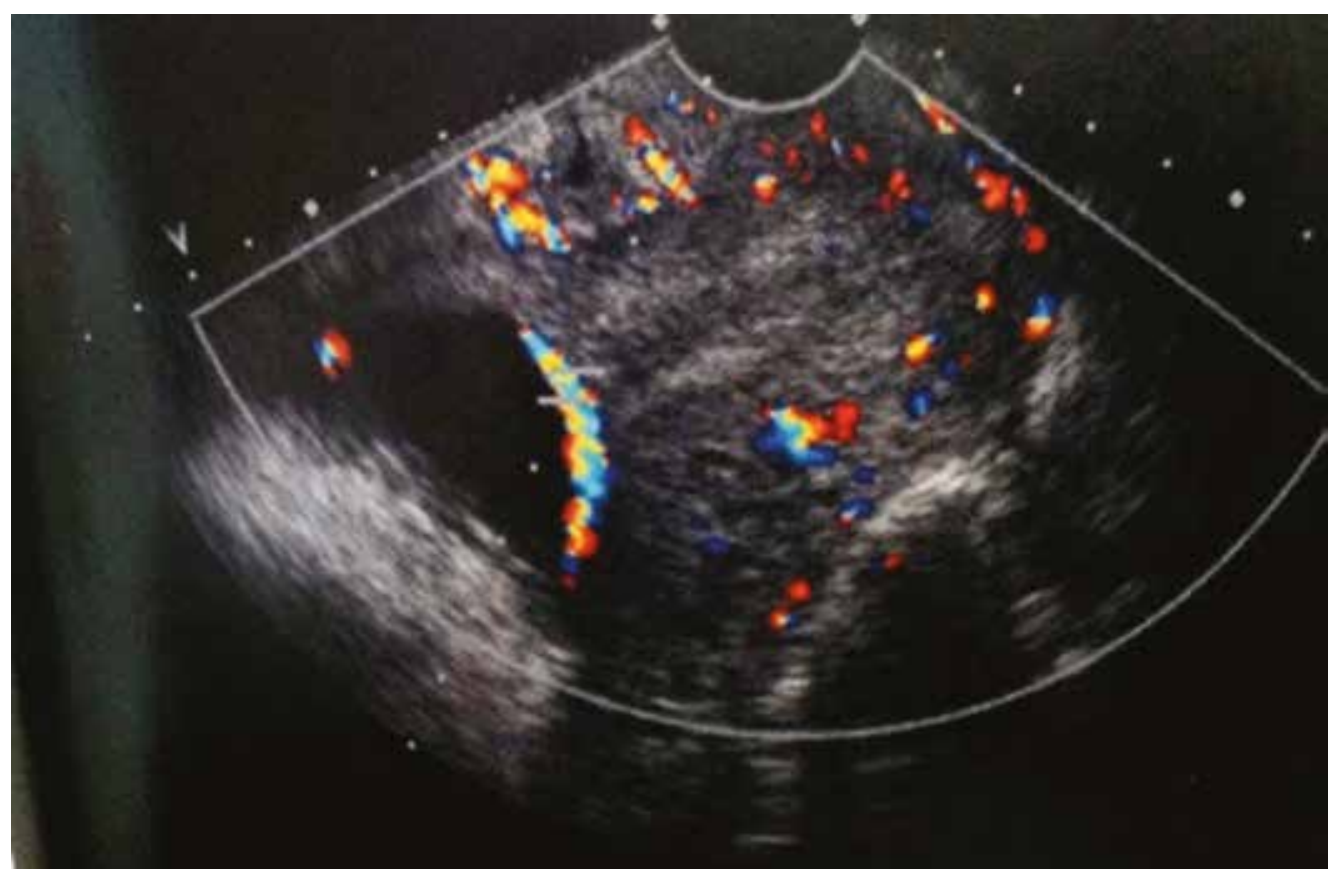

Figura 1. Imagen de la ecografía transvaginal de la paciente en modo 2D aplicando doppler color que permite ver vasos sanguíneos fijos previos al cérvix (Unidad de Ecografía, HMUA de Envigado) 
les; las pérdidas hemáticas maternas cuantificadas en la cesárea fueron de 1300 cc, con estabilidad hemodinámica y control de hemoglobina materna de 9,5 g/dl. La paciente estuvo hospitalizada por 3 días y no presentó ninguna complicación posoperatoria temprana o tardía.

Aspectos éticos. Se solicitó autorización a la paciente para publicar la revisión de la historia clínica y la imagen ecográfica. Se garantizó la confidencialidad de la información para proteger los derechos de la paciente.

\section{MATERIALES Y MÉTODOS}

A partir de las preguntas sobre la patogénesis de la vasa previa, la exactitud de la ecografía doppler utilizada para el diagnóstico y el pronóstico perinatal de los fetos afectados con vasa previa se hizo una búsqueda en la base de datos Medline vía PubMed con los términos: "vasa previa", "ultrasonography” y “prognosis" para artículos con estudios publicados entre 1993 y 2013 en inglés y español, se incluyeron artículos de revisión de tema, revisiones sistemáticas y reporte de casos.

\section{RESULTADOS}

Se encontraron 35 artículos, se analizaron todas las referencias y se encontró que 22 corresponden a reportes de caso, 8 a revisiones de tema y 5 a revisiones sistemáticas.

Patogénesis. Las dos principales causas asociadas a vasa previa son: a) la inserción velamentosa del cordón umbilical, y b) la presencia de vasos que pasan entre lóbulos de la placenta, como en el caso de placentas bilobuladas o cotiledones aberrantes $(3,21)$. En el primer caso, Sepúlveda et al. (22) informan que si bien en aproximadamente un 90\% de los casos la inserción del cordón umbilical ocurre en el centro de la placenta, en el 10\% restante la inserción es marginal, es decir, a menos de 1-2 cm del borde placentario, y en el $1 \%$ de los casos la inserción ocurre en las membranas ovulares fuera de la masa placentaria (inserción velamentosa). Este autor analizó 832 embarazos únicos usando ultrasonido y doppler color 2D y 3D encontrando 8 casos de vasa previa, 7 asociados a inserción velamentosa y una inserción excéntrica del cordón (22). Hasegawa et al. (23) analizaron 3446 embarazos con el objetivo de determinar el sitio de la inserción del cordón umbilical, encontrando 40 casos de inserción velamentosa del cordón, de los cuales 25 estaban asociados a vasa previa.

La segunda causa asociada tiene relación con la presencia de placentas bilobuladas y cotiledones aberrantes, que pueden tener vasos sanguíneos que crucen entre uno o más lóbulos accesorios a nivel del segmento uterino inferior (3). En orden de frecuencia de las anormalidades placentarias tenemos la placenta bilobulada, multilobar, accesoria y de ubicación marginal, estos hallazgos no son excluyentes entre sí, y nos llevan a pensar que el fenómeno de vasa previa surgiría como un defecto en las primeras fases del desarrollo placentario (24). La placenta bilobulada tiene una incidencia de 0,04 a 4,2\%, y en dos tercios de los casos se asocia a inserción velamentosa del cordón y tiene un riesgo mayor de retención de placenta (24, 25). Lee et al. analizaron 93.874 embarazos, de los cuales 18 tenían diagnóstico de vasa previa, de estos a 17 se les hizo el diagnóstico prenatal por ultrasonido, 10 casos estaban asociados a cordón velamentoso, 3 a placenta bilobulada, 2 a placenta succenturiate y 2 a placenta marginal (26).

Se ha descrito que el embarazo múltiple y la fertilización in vitro se han asociado a una mayor frecuencia de vasa previa. Respecto al primero, el estudio de Oyelese et al. encontraron 7 embarazos gemelares en 155 casos de vasa previa, lo que representa 4,5\%, siendo este mayor al de la población general (27); Raga reporta un 10\% de vasa previa en embarazos gemelares (28). Se ha reconocido que embarazos concebidos por técnicas de fertilización in vitro (FIV) tienen un riesgo del 10\% de presentar vasa previa (29); en un estudio de 100 placentas de embarazos con FVI, 14 casos tenían inserción velamentosa del cordón (30); Schachter et al. encontraron una incidencia de vasa previa en su institución de 1 en 293 partos de FVI, comparadas 
con una tasa de vasa previa de 1 en 6.068 partos normales, este predominio era superior al de la población general (31).

Diagnóstico ecográfico. En 1990, Nelson et al. señalaron la utilidad del Doppler-color para el diagnóstico de vasa previa en un embarazo de 26 semanas (15). Solo en 1996 se comunicó el primer diagnóstico de vasa previa durante el examen rutinario de segundo trimestre, lo que determinó una cesárea electiva a las 35 semanas que resultó en un recién nacido sano (16). En el primer trimestre la ultrasonografía no sería útil porque en su mayoría el trofoblasto cubre una gran extensión de la superficie uterina; sin embargo, durante el examen que se realiza entre las 11-14 semanas es posible identificar con precisión el sitio de inserción del cordón umbilical en la placenta (22). La técnica incluye la realización de ultrasonido con modo doppler color, aplicado sobre el orificio cervical interno que permita identificar vasos sanguíneos que discurran de forma anómala a este nivel (21), se puede realizar por vía transvaginal, transperineal y transabdominal, siendo la primera la más utilizada (26). Oyelese et al. (27) analizaron 155 casos de vasa previa, donde se describe que en 95 casos $(62,1 \%)$ se encontraba una placenta de inserción baja en el segundo trimestre y al momento del parto solo 31 casos (20,3\%), observando con esto una resolución en dos de cada tres casos (27). Este hallazgo también fue descrito por Nomiyama et al., quienes reportaron que de dos casos diagnosticados antes del parto solo uno persistió hasta el final del embarazo (32). No es claro si corresponden a falsos positivos o a resolución espontánea de la anormalidad del cordón.

$\mathrm{Al}$ analizar los diferentes estudios con respecto a la utilidad de la ecografía para el diagnóstico de vasa previa contamos con el primer estudio de Nomiyama donde el objetivo fue identificar la inserción del cordón umbilical, inserción velamentosa del cordón y vasa previa; se tomaron 587 casos (555 embarazos únicos y 16 embarazos gemelares), la edad gestacional al diagnóstico fue 18-20 semanas, utilizaron de rutina doppler color en ecografía transvaginal y transabdominal e identificaron la inserción del cordón umbilical en 580 de los 581 casos (99,8\%), en uno no fue visto. El estudio describe en la inserción velamentosa del cordón, para el doppler, una sensibilidad del 100\% (5/5) y una especificidad del 99,8\% (580/581) (32). Catanzarite et al. (29) analizaron 33.208 embarazos con el objetivo de determinar la especificidad del diagnóstico ultrasonográfico antenatal de vasa previa, la edad gestacional del diagnóstico fue entre las 20-24 semanas (67\%), utilizando ecografía transvaginal y transabdominal con doppler color o doppler poder. El estudio informa una especificidad del $91 \%$ (29). Estos autores consideran que existen algunas circunstancias que incrementan la frecuencia de falsos negativos, tales como la dirección y ubicación baja de los vasos sanguíneos, la obesidad materna, la presencia de cicatrices abdominales previas y vejiga llena; además sugiere tener en cuenta los siguientes diagnósticos diferenciales: separación de membrana corio-amniótica, circular de cordón normal, seno vascular marginal placentario y una banda amniótica.

Pronóstico. La principal complicación de la vasa previa es su ruptura, lo que lleva a una hemorragia fetal aguda, choque hipovolémico, asfixia fetal y muerte perinatal (33). El pronóstico es sustancialmente mejor cuando el diagnóstico se hace en el periodo prenatal; la prevalencia de asfixia perinatal y anemia neonatal severa es elevada en casos sin diagnóstico prenatal. Oyelese informa que el puntaje de Apgar promedio fue de 8 y 9 cuando se contó con el diagnóstico prenatal y solo de 1 y 4 en los casos de sobrevivientes sin diagnóstico previo; además, el 58\% de los recién nacidos sin diagnóstico previo requirió transfusión sanguínea, mientras esta se requirió solo en un $3 \%$ de los recién nacidos en los cuales se contaba con diagnóstico prenatal. Si el diagnóstico no es prenatal la mortalidad perinatal es de aproximadamente un $60 \%$ comparada con un $3 \%$ en que se hizo el diagnóstico (27). Fung et al. analizaron 53 casos con diagnóstico de vasa previa entre 1980 y 1997; en el grupo sin diagnóstico prenatal, 8 casos tuvieron puntaje de APGAR 
a los 5 minutos menor de 7, y en los que tenían diagnóstico antenatal solo uno tuvo un puntaje de APGAR menor de 7 a los 5 minutos al nacer, en el grupo sin diagnóstico antenatal de vasa previa 12 tenían anemia aguda que requirió transfusión, y en el grupo con diagnóstico antenatal solo uno requirió transfusión. Estos autores evaluaron también las consecuencias en la madre, 20 presentaron sangrado intraparto, mientras que en el grupo en los cuales se realizó el diagnóstico prenatal (22 pacientes) solo 6 presentaron sangrado vaginal antes del parto; por otra parte informan que de 31 fetos en los que el diagnóstico se realizó durante o después del parto 2 murieron como causa de sangrado, la mortalidad fetal en este grupo fue de 22,5\%; en el grupo en el cual se realizó el diagnóstico prenatal (22 pacientes), no hubo ninguna muerte neonatal (2). Lee et al. encontraron asociación entre hemorragia vaginal anteparto en 6 de los 18 casos reportados de vasa previa (33\%) (26); Hasegawa et al. (23) analizaron 340 casos con el fin de identificar la inserción baja del cordón umbilical en el primer trimestre (9-11 semanas) encontrando 35 casos (tercio inferior segmento uterino), de los cuales 4 tenían inserción velamentosa del cordón y 4 placenta accesoria como patogénesis de vasa previa. Como complicaciones asociadas encontró 9/35 casos de ruptura prematura de membranas (25\%), 4/35 casos pequeños para edad gestacional (11\%) y 2/35 casos con necesidad de cesárea de urgencias (5,7\%) (23); Nomiyana et al. (32), al igual que los autores anteriores, también encontraron asociación de vasa previa con ruptura prematura de membranas. No se ha informado aún ningún riesgo aumentado de recurrencia de vasa previa en los siguientes embarazos (3).

\section{CONCLUSIÓN}

La vasa previa es una complicación obstétrica poco frecuente. La evaluación de la placenta por ultrasonido es un método útil para determinar la inserción del cordón umbilical y sus posibles anormalidades. El pronóstico es pobre, con índices altos de mortalidad fetal si no se realiza un diagnóstico antenatal.

\section{REFERENCIAS}

1. Evans GM. Vasa praevia. Br Med J. 1952;2:1243.

2. Fung TY, Lau TK. Poor perinatal outcome associated with vasa previa: is it preventable? A report of three cases and review of the literature. Ultrasound Obstet Gynecol. 1998;12:430-3.

3. Derbala Y, Grochal F, Jeanty P. Vasa previa. J Prenat Med. 2007;1:2-13.

4. Lobstein J. Archives de L'art des Accouchements 1801; Strasbourg; p. 320.

5. Gianopoulos J, Carver T, Tomich PG, Karlman R, Gadwood K. Diagnosis of vasa previa with ultrasonography. Obstet Gynecol. 1987;69:488-91.

6. Carp HJ, Mashiach S, Serr DM. Vasa previa: a major complication and its management. Obstet Gynecol. 1979;53:273-5.

7. Dougall A, Baird CH. Vasa previa - report of threecases and review of the literature. Br J Obstet Gynaecol. 1987;94:712-5.

8. Naftolin F, Mishell DR Jr. Vasa previa: report of 3 cases. Obstet Gynecol. 1965;26:561-5.

9. Antoine C, Young BK, Silverman F, Greco MA, Alvarez SP. Sinusoidal fetal heart rate pattern with vasa previa in twin pregnancy. J Reprod Med. 1982;27:295-300.

10. Curl CW, Johnson WL. Vasa previa, antepartum diagnosis. Obstet Gynecol. 1968;31:328-30.

11. Oyelese KO, Turner M, Lees C, Campbell S. Vasa previa: an avoidable obstetric tragedy. Obstet Gynecol Surv. 1999;54:138-45.

12. Browne AD, Brennan RK. The application, value, and limitations of amnioscopy. J Obstet Gynaecol Br Commonw. 1968;75:616-21.

13. Barham KA. Amnioscopy-amniotomy: a look at surgical induction of labor. Am J Obstet Gynecol. 1973;117:35-8.

14. Odunsi K, Bullough CH, Henzel J, Polanska A. Evaluation of chemical tests for fetal bleeding from vasa previa. Int J Gynaecol Obstet. 1996;55:207-12.

15. Abnormalities of placentation. En: Cunningham FG, Wiliam JW, editors. Williams obstetrics. 21 st ed. New York: McGraw-Hill; 2001. p. 828-34.

16. Benedetti TJ. Obstetric hemorrhage. En: Gabbe SG, Niebyl JR, Simpson JL, editors. Obstetrics: normal and 
problem pregnancies. 4th ed. New York: ChurchillLivingstone; 2002. p. 503-38.

17. Apt L. Melena neonatorum. J Pediatr. 1955;47:1-5.

18. VanDrie DM, Kammeraad LA. Vasa previa: case report, review and presentation of a new diagnostic method. J Reprod Med. 1981;26:577-80.

19. Nimmo MJ, Kinsella D, Andrews HS. MRI in pregnancy; the diagnosis of vasa previa by magnetic resonance imaging. Bristol Med Chir J. 1988;103:12.

20. Clerici G, Burnelli L, Lauro V, Pilu GL, Di Renzo GC. Prenatal diagnosis of vasa previa presenting as amniotic band. A not so innocent amniotic band. Ultrasound Obstet Gynecol. 1996;7:61-3.

21. Daly-Jones E, John A, Leahy A, McKenna C, Sepúlveda W. Vasa praevia; a preventable tragedy. Ultrasound. 2006;16:8-14

22. Sepulveda W. Velamentous insertion of the umbilical cord: a first trimester sonografic screening study. J Ultrasound Med. 2006;25:963-8.

23. Hasegawa J, Matsuoka R, Ichizuka K, Sekizawa A, Farina A, Okai T. Velamentous cord insertion into the lower third of the uterus is associated with intrapartum fetal heart rate abnormalities. Ultrasound Obstet Gynecol. 2006;27:425-9.

24. Benirschke K, Kaufmann P. Pathology of the human placenta. 4th ed. New York: Springer-Verlag; 2000. p. 353-9, 401-4.

25. Fox H. Pathology of the placenta. 2nd ed. Philadelphia: WB Saunders; 1997. p. 64-5.
26. Lee W, Lee VL, Kirk JS, Sloan CT, Smith RS, Comstock CH. Vasa praevia: prenatal diagnosis, natural evolution, and clinical outcome. Obstet Gynecol. 2000;95:572-6.

27. Oyelese Y, Catanzarite V, Prefumo F, Lashley S, Schachter M, Tovbin Y, et al. Vasa Previa: The impact of prenatal diagnosis on outcomes. Am College Obstet Gynecol. 2004;5:937-42.

28. Raga F, Ballester MJ, Osborne NG, Bonilla-Musoles F. Role of color flow Doppler ultrasonography in diagnosing velamentous insertion of the umbilical cord and vasa previa. A report of two cases. J Reprod Med. 1995;40:804-8.

29. Catanzarite V, Maida C, Thomas W, Mendoza A, Stanco L, Piacquadio KM. Prenatal sonographic diagnosis of vasa previa: ultrasound findings and obstetric outcome in ten cases. Ultrasound Obstet Gynecol. 2001;18:109-15.

30. Englert Y, Imbert MC, van Rosendael E, Belaisch J, Segal L, Feichtinger W, et al. Morphological anomalies in the placentae of IVF pregnancies: preliminary report of a multicentric study. Hum Reprod. 1987;2:155-7.

31. Schachter M, Tovbin Y, Arieli S, Friedler S, Ron-El R, Sherman D. In vitro fertilization is a risk factor for vasa previa. Fertil Steril. 2002;78:642-3

32. Nomiyama M, Toyota Y, Kawano H. Antenatal diagnosis of velamentous umbilical cord insertion and vasa previa with color Doppler imaging. Ultrasound Obstet Gynecol. 1998;12:426-9.

33. Robert JA, Sepúlveda W. Fetal exsanguination from ruptured vasa previa: still a catastrophic event in modern obstetrics. J Obstet Gynaecol. 2003;23:574. 